\title{
FORMA TUMORAL DA NEUROCISTICERCOSE: EXERESE DE CISTICERCO DE 70X77 MM E TRATAMENTO COM PRAZIQUANTEL
}

\author{
RELATO DE CASO
}

\author{
BENEDICTO OSCAR COLLI \\ JOAO ALBERTO ASSIRATI JR. \\ NILTON ELUARDO GUERREIRO
}

\author{
NELSON MARTELLI \\ HELIO RUBENS MACHADO \\ ANGELA BWLLUCI
}

O acometimento cerebral pela cisticercose apresenta grande importância em nosso melo nao so pela moroiaade e mortaliaade elevadas, mas tamoem pela alta freqüëıcıa dessa parasitose. Entre suas diferentes manirestações clínicas destaca-se a uipertensäo intracraniana, pela sua graviääde $1,3,12,25,26$ e potencialidade cirúrgica. No Hospital das Clínicas da Faculdade de Medicina de Ribeirão Preto a neurocisticercose foi cbservada em 2.7\% dos pacientes atendidos por apresentarem afecções neurológicas e a hipertensão intracraniana pura ou combinada com outra sintomatologia, foi observada em $35,8 \%$ destes casos ${ }^{41}$. A presença de cisticercos que atingem tamanhos suficientes para se comportarem como processos expansivos na região supratentorial é descrita na literatura 4,6,10,18,19,20,28,36,40 e a demonstração destes cisticercos tornou-se mais objetiva com o uso da tomografia computadorizada (TC) 6,7,8,10,14,20,21,22,24,28,29,30,42.

O caso relatado apresenta como peculiaridade, além do diagnóstico diferencial com neoplasia, o enorme volume do cisto e a associação com outras vesículas menores, esparsas no parênquima e demonstradas pela TC que levaram à combinação dos tratamentos cirúrgico e clínico.

\section{OBSERVAÇAO}

MHSO, 22 anos, branca, procedente de Miguelópolis-SP. Data da internação 4-setembro-1982. Apresentou episódio de crise convulsiva lateralizada à direita há 5 meses, seguido de perda da força neste dimídio, que regrediu parcialmente. Apresentou mais um episódio semelhante há dois meses, a partir do qual desenvolveu dificuldade progressiva para falar. Há 20 dias está apresentando incontinência urinária. Veio encaminhada de outra cidade com diagnóstico de tumor intracraniano à esquerda, inoparável (sic); medicada com Gardenal $100 \mathrm{mg} /$ dia. Exame Neurológico - Disfásica, marcha parética, hemiparesia completa à direita com predominio braquio-facial, hipertonia $e$ hiperreflexia profunda à direita, edema de papila bilateral. Hipótese diagnóstica -

Departamento de Cirurgia, Ortopedia e Traumatologia (Disciplina Neurocirurgia) da Faculdade de Medicina de Ribeirão Preto da Universidade de São Paulo. Trabalho apresentado no XX Congresso Latinoamericano de Neurocirurgia (São Paulo, 1983). 
Processo expansivo de hemisfério cerebral esquerdo. Exames complementares - TC (06-09-82) - tumoração cística $(70 \times 77 \mathrm{~mm})$ de contornos bem definidos, hipodensa, localizada em área fronto-parieto-temporal esquerda, apresentando efeito de massa, deslocando a linha média e o ventrículo lateral esquerdo. Presença de outras pequenas formações arredondadas hipodensas, dispersas no parênquima cerebral (Fig. 1); HD-Neurocisticercose? Carotido-angiografia (06-09-82) - processo expansivo avascular fronto-parieto-temporal esquerdo. Cirurgia (06-09-82) - craniotomia fronto-parieto-temporal esquerda: enorme cisto subcortical que aflorava à superfície por entre dois giros corti-

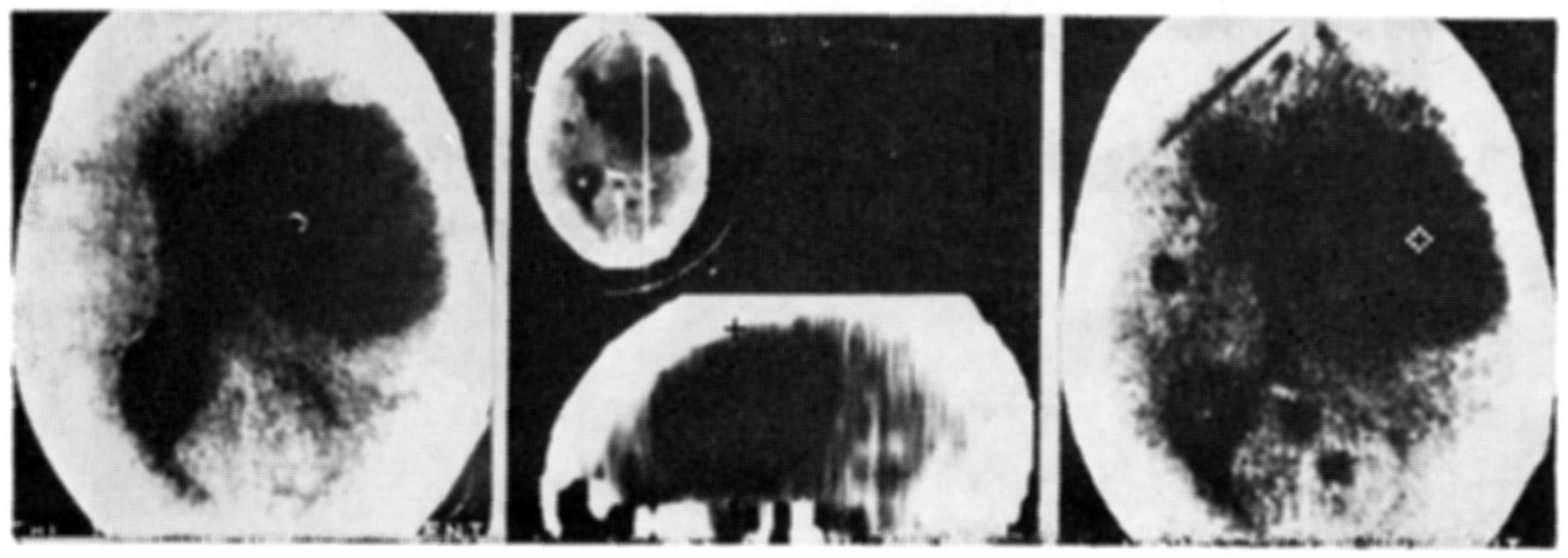

Fig. 1 - Caso M.H.S.O.: tomografia computadorizada do cranio. a esquerda: grande cisto na regiáo fronto-parieto-temporal esquerda ocasionando acentuado desvio da linha média e colabamento do ventriculo homolateral. No meio: reconstrucão para-sagital mostrando em detalhe o grande cisto. A direita: corte mais candal mostrando várias dreas hipodensas menores espalhadas no parenquima, além do cisto maior.

cais. O cisto foi dissecado e parcialmente expulso para fora do parênquima; posteriormente rompeu-se e duas vesículas $(30 \times 30 \mathrm{~mm})$ sairam integras do seu interior. A parede restante foi removida através de dissecção microcirúrgica e não foi encontrado escólex (cisticerco racemoso). Foi observado outro cisto típico de cisticerco na região parietal posterior, de $10 \times 10 \mathrm{~mm}$, que foi removido para exame. O exame histopatológico da membrana foi compatível com membrana de cisticerco. Evolução pós-operatória - evoluiu bem, com regressão completa do quadro neurológico, exceto o edema de papila que diminuiu parcialmente, tendo alta em 19-09-82. Reinternação (22-10-82) - submetida a tratamento com praziquantel durante 21 dias $(50 \mathrm{mg} / \mathrm{kg} /$ dia). Exame Neurológico edema de papila incipiente. Exames Complementares - Liquido Cefalorraqueano (LCR) em 25-10-82: células 0,3 por mm3, proteínas $15 \mathrm{mg} \%$, glicose $59 \mathrm{mg} \%$, cloretos $766 \mathrm{mg} \%$, reação de fixação de complemento para cisticercose (RFCC) negativa; LCR (01-11-82): células 109,3 por $\mathrm{mm3}$ (43\% de linfócitos, $13 \%$ de neutrófilos, $4 \%$ de eosinófilos e $1 \%$ de monócitos), proteínas $23 \mathrm{mg} \%$, glicose $53 \mathrm{mg} \%$, cloretos $743 \mathrm{mg} \%$, RFCC reagente fraca; LCR (17-11-82): células 3 por $\mathrm{mm} 3$, proteínas $10 \mathrm{mg} \%$, glicose $55 \mathrm{mg} \%$, cloretos $772 \mathrm{mg} \%$, RFCC negativa; TC (25-11-82): várias formações cisticas no parênquima cerebral, com diametro de 10 e $30 \mathrm{~mm}$, predominando em áreas fronto-parietais, algumas com halo de captação ao redor inclusive na área da intervenção círúrgica; dilatação do corno frontal do ventrículo lateral esquerdo (Fig. 2); RX Crânio (25-11-82): normal. Evolução - durante e após o periodo de tratamento evoluiu sem intercorrenclas. vltimo 
retorno em 09-03-83 sem queixas e com exame neurológico normal. TC (25-02-83): área hipodensa de $20 \mathrm{~mm}$ na região fronto-parietal esquerda, no local da extirpação cirúrgica do grande cisto; dilatação do ventrículo lateral esquerdo; pequenos cistos com retencão de contraste no hemisfério cerebral esquerdo e pequenas calcificaçóes no parênquima (Fig. 3).
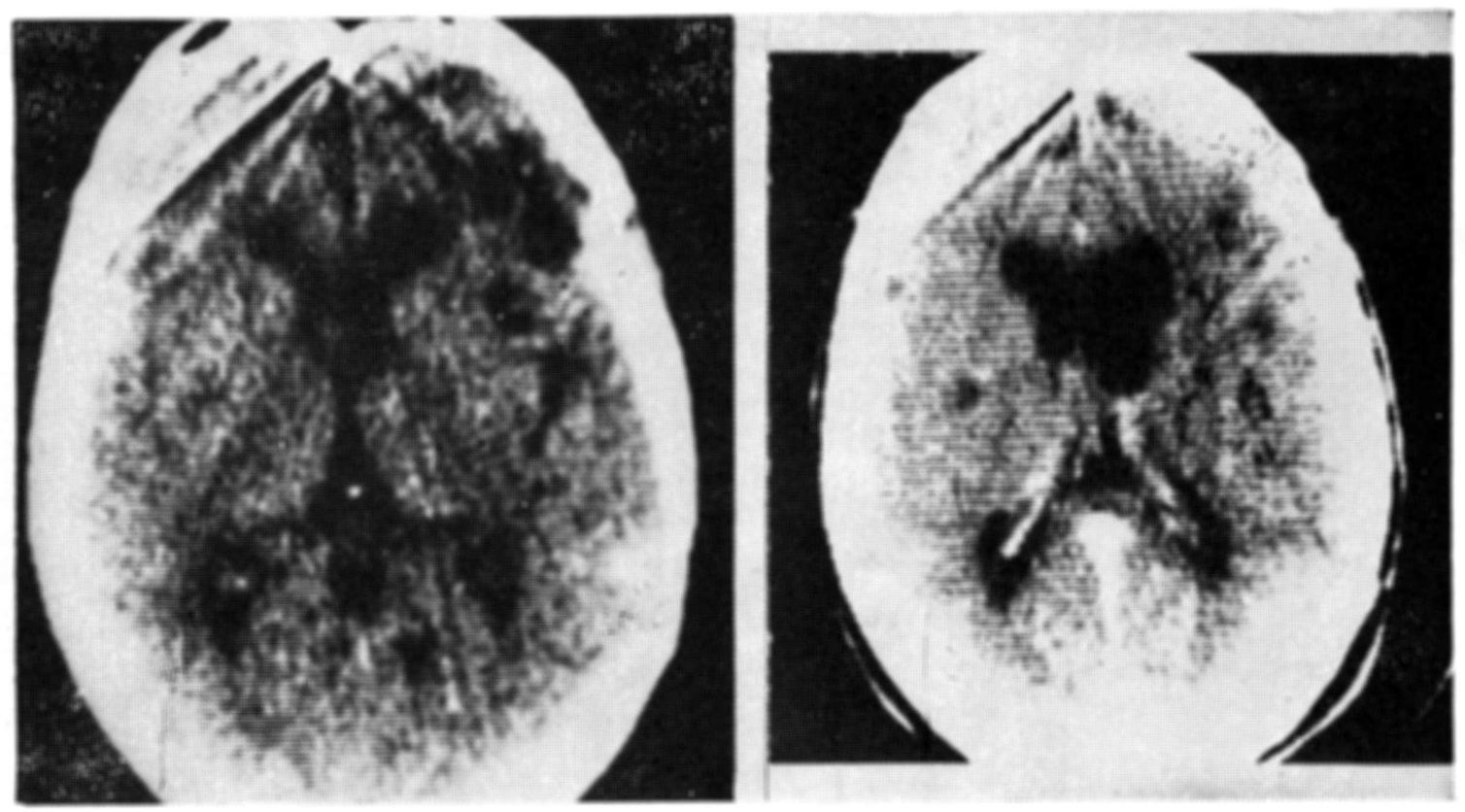

Fig. 2 - Caso M.H.S.O.: tomografia computadorizada realizada 1 mês após a cirurgia evidenciando areas hipodensas no parênquima, inclusive na área operada, $e$ dilatando $o$ ventriculo lateral esquerdo.

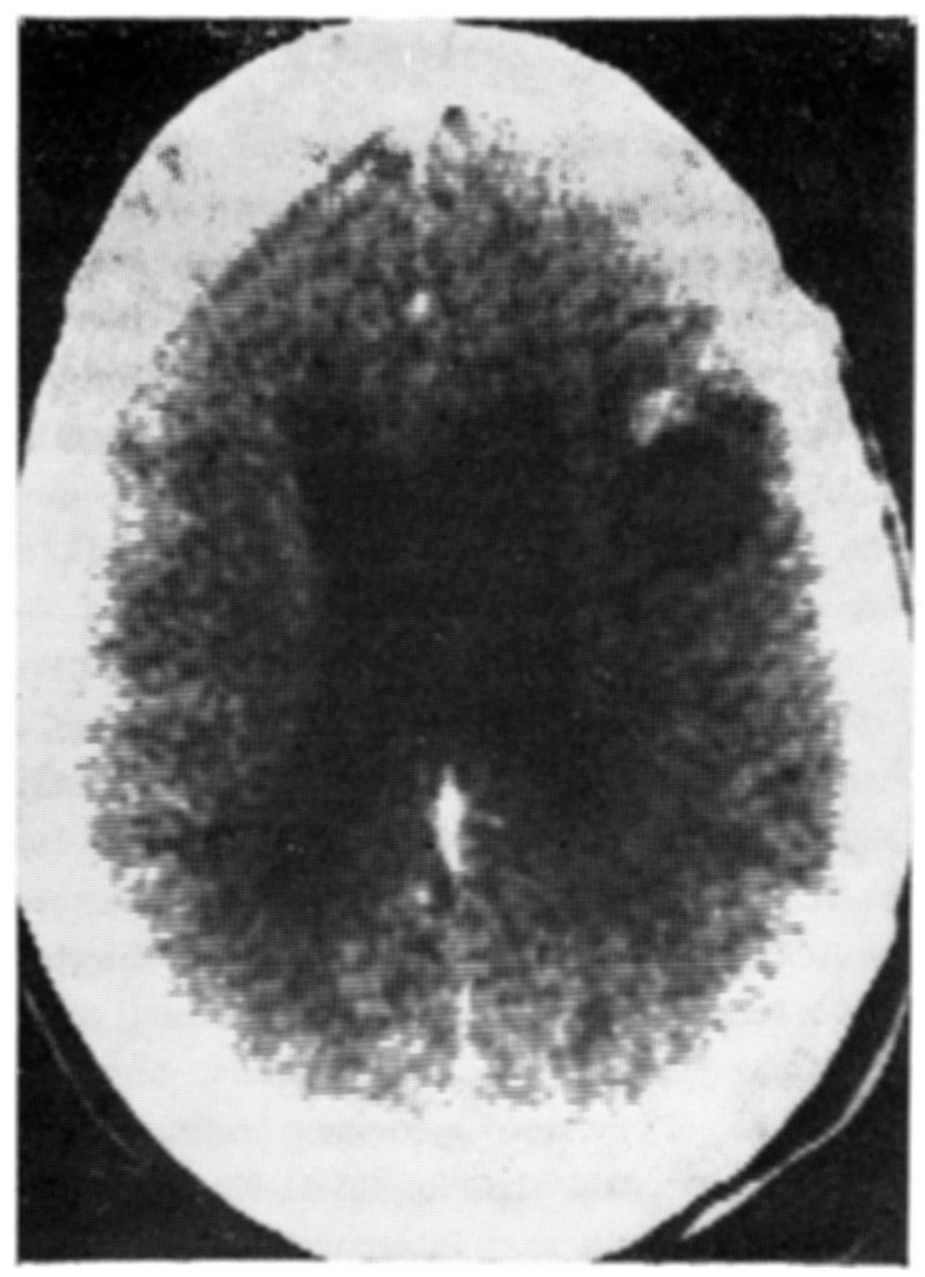

Fig. 3 - Caso M.H.S.O.: tomografia computadorizada realizada 3 meses apos o tratamento com praziquantel, evidenciando área hipodensa no local operado, com retensão de contraste circunscrita a pequena area de sua periferia e dilatacão do ventriculo homolateral. 


\section{COMENTARIOS}

A forma hipertensiva da neurocisticercose, na maioria dos casos, necessita de tratamento cirúrgico, quer seja sob a forma de exéreses de grandes cistos que se comportam como processos expansivos intracranianos ou de cisticercos alojados no sistema ventricular, quer seja sob a forma de derivações do LCR. Nos casos em que ocorrem obstruções do sistema ventricular e hidrocefalia, o diagnóstico diferencial se impõe com neoplasias, geralmente da fossa posterior. Quando o cisticerco ocupa espaço na região supratentorial, o diagnóstico diferencial deve ser feito com qualquer tumor, senso lato, dessa região.

No diagnóstico diferencial, de grande valia é o exame do LCR que pode fornecer o diagnóstico de certeza da neurocisticercose em vida, nos casos em que reação de fixação de complemento para cisticercose é positiva. Quando por algum motivo, com sinais de hipertensão intracraniana grave, a obtenção de amostras do LCR por punção lombar ou suboccipital para exame é difícil ou, então, a reação de fixação de complemento é negativa nas amostras colhidas ${ }^{12,18}$, o problema do diagnóstico diferencial com neoplasias torna-se complicado. Eventualmente, a observação de calcificações típicas de um cisticerco no RX simples de crânio pode orientar no diagnóstico $2,3,9,10,11,17,23,24,25,30,40$. As angiografias mostram sinais de processos expansivos avasculares, o que não auxilia muito no diagnóstico diferencial 12,17,24,25,27.

Os achados ventriculográficos referidos por vários autores 2,3,5,10,11,12,13,16, 17,22,40,42 nem sempre são suficientes para se estabelecer o diagnóstico de neurocisticercose. A utilização de contrastes iodados, especialmente os hidrossolúveis, possibilitou a delineação perfeita de vesículas intraventriculares, geralmente móveis $3,5,13,16,40,42$ que nas regiões em que a parasitose é freqüente pode ser considerada suficiente para o seu diagnóstico 13. A TC os cisticercos localizados no parênquima encefálico podem apresentar-se como áreas arredondadas hipodensas, de tamanho variado, com ou sem retenção de contraste na periferia, ou então

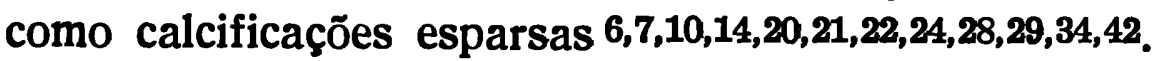

No caso relatado, após a realização da TC foi aventada a hipótese diagnóstica de cisticercose cerebral devido à presença de um grande cisto $(70 \times 77 \mathrm{~mm})$ sem áreas de retenção de contraste, com conteúdo líquido de densidade semelhante à do LCR, associado a outras áreas hipodensas menores, distribuídas no parênquima; a estes dados acrescentou-se o fato de se estar em zona endêmica dessa parasitose.

A indicação da exérese cirúrgica do cisto maior foi devida ao seu grave efeito de massa, observado na TC e angiografia. Curiosamente, apesar da ruptura do cisto durante a dissecção, teve-se a impresão de que ele foi removido completamente, mas a TC de controle realizada dors meses e meio após a cirurgia mostrou área hipodensa no local da cirurgia, com região de captação que foi interpretada como um excólex.

Em nosso caso, devido à remissão completa da sintomatologia neurológica e à concomitância de outras lesões no parênquima, a paciente foi submetida a tratamento com praziquantel. Durante este período observou-se no LCR reação 
celular eosinofílica e positivação da reação de fixação de complemento para a cisticercose, ambas transitórias. $O$ controle tomográfico realizado três meses após o tratamento evidenciou melhora do quadro com o desaparecimento dos cistos no parênquima. No local operado foi notada área hipodensa de $20 \mathrm{~mm}$, interpretada como provável seqüela cirúrgica, e uma dilatação no corno frontal do ventrículo lateral esquerdo.

O tratamento da neurocisticercose com praziquantel (pirazino isoquinoleína), tem sido empregado há alguns anos no Brasil, Chile, Colômbia, Equador e México $8,15,31,32,33,35,37,38,39$. Os resultados obtidos podem ser considerados muito bons (64 a $87 \%$ de curas e' melhoras) dependendo da forma clínica considerada, observando-se também o aparecimento de reações no LCR típicas da doença, transitórias, que representam o efeito da destruição do cisticerco pela medicação. $O$ uso do praziquantel veio contribuir de forma bastante favorável no tratamento dos pacientes com neurocisticercose, podendo ser uma esperança de cura, relativamente frequiente, em contraste com os baixos índices de cura observados com tratamentos cirúrgicos, geralmente paliativos $\mathbf{3 2}$.

\section{RESU:MO}

$O$ caso descrito refere-se a paciente do sexo feminino, com 22 anos, com história de dois episódios de crise convulsiva lateralizada à direita, hemiparesia direita e disfasia. O exame neurológico mostrou ainda edema de papila bilateral. A tomografia computadorizada (TC) do crânio mostrou um cisto de $70 \times 77 \mathrm{~mm}$, ocupando a região fronto-parieto-temporal esquerda, ocasionando grande desvio da linha média, além de outras lesões menores, semelhantes, esparsas no parênquima. Com o diagnóstico de neurocisticercose foi submetida a craniotomia, sendo removidos o cisto maior (cisticerco racemoso) e pequeno cisticerco cortical $(10 \times 10 \mathrm{~mm})$ para exame. Teve alta 13 dias após a cirurgia, com regressão completa do quadro neurológico, exceto o edema de papila (regressão parcial). Um mês após foi submetida a tratamento com praziquantel (50 $\mathrm{mg} / \mathrm{kg} /$ dia durante 20 dias). O exame de LCR foi normal antes deste tratamento e, durante ele, apresentou reação celular eosinofílica, com positivação da reação de fixação de complemento para a cisticercose, ambas transitórias. A TC prévia ao tratamento mostrou várias áreas hipodensas de 10 a $40 \mathrm{~mm}$, esparsas no parênquima, inclusive na área operada. A TC realizada três meses após mostrou diminuição do número de cistos do parênquima, persistindo apenas uma área hipodensa de $20 \mathrm{~mm}$ no local operado e dilatação do corno frontal do ventrículo do mesmo lado. A evolução clínica foi excelente e o exame neurológico foi normal 3 meses depois.

\section{SUMMARY}

Tumoral form of cerebral cysticercosis: surgical removal of a cysticercus $(70 \times 77 \mathrm{~mm})$ and clinical treatment with praziquantel: case-related.

The authors report a case of a female patient, age 22, who presented episodes of focal seizures, right hemiparesis and dysphasia of five months duration. The 
neurologic exam revealed bilateral papilledema and computarized tomography of the head showed a cystic lesion $(70 \times 77 \mathrm{~mm})$ in the left fronto-temporo-parietal area, midline shift and similar lesions scattered in the brain parenchyma. She was submitted to a left craniotomy with the diagnosis of cerebral cysticercosis and the major cyst (Cysticercus racemosus) and a small cortical cyst $(10 \times 10$ $\mathrm{mm}$ ) were removed to aliviate the increased intracraneal pressure and for histopathological examination. Thirteen days after surgery she was discharged without neurological deficits, except mild papilledema. One month later she was treated with praziquantel $(50 \mathrm{mg} / \mathrm{kg} /$ day $)$ during 21 days. The CSF examination was normal before the treatment started and showed a transient eosinophilic cellular reaction with positivation of the complement fixation test for cysticercosis while on treatment. The CT-Scan performed before the medical treatment revealed ventricular dilatation and several low density areas (10 to $40 \mathrm{~mm}$ ) scattered in the cerebral parenchyma, including the operative site. CT-Scan performed three months later showed small low density areas only in the left cerebral hemisphere, including the operative site, calcification in the parenchyma, and slight dilatation of the left lateral ventricle. The clinical evolution was uneventfull and the neurologic examination was normal three months later.

\section{REFERENCIAS}

1. ALMEIDA, G. M.; PEREIRA, W. C. \& FACURE, N. O. - Ventriculo-auriculostomia nos bloqueios ao trânsito do líquido cefalorraqueano na cisticercose encefálica. Arq. Neuro-Psiquiat. (São Paulo) 24:163, 1966.

2. ARANA, R. \& ASENJO, A. - Ventriculographic diagnosis of cysticercosis of the posterior fossa. J. Neurosurg. 2:181, 1945.

3. ARSENI C. \& SAMITCA, D. C. - Cysticercosis of the brain. Brit. med. J. $5043: 494,1957$.

4. ASSIS, J. L. \& TENUTO, R. A. - Cisticerco racemoso intraventricular. Extirpação cirúrgica. Arq. Neuro-Psiquiat. (São Paulo) 6:247, 1948.

5. BALAPARAMESWARARAO, S. \& DINAKAR, I. - Ventriculographic features of cerebral cysticercosis. Brit. J. Radiol. 43:267, 1970.

6. BERMAN, J. D.; BEAVER, P. C.; CHEEVER, A. W. \& QUINDLEN, E. A. Cysticercus of 60-milliliter volume in human brain. Amer. J. trop. Med. Hyg. 30:616, 1981.

7. BENTSON, J. R.; WILSON, G. H.; HELMER, E. \& WINTER, J. - Computed tomography in intracranial cysticercosis. J. comp. assist. Tomogr. 1:464, 1977.

8. BOTERO, D. \& CASTANO, S. - Treatment of cysticercosis with praziquantel in Colombia. Amer. J. trop. Med. Hyg. 31:810, 1982.

9. CANELAS, H. M. - Neurocisticercose: incidência, diagnóstico e formas clínicas. Arq. Neuro-Psiquiat. (Săo Paulo) 20:1, 1962.

10. CARBAJAL, J. R.; PALACIOS, E.; AZAR-KIA, B. \& CHURChILL, R. - Radiology of cysticercosis of the central nervous system including computed tomography. Radiology 125:127, 1977.

11. CARDENAS Y CARDENAS, J. - Cysticercosis II. Pathologic and radiologic findings. J. Neurosurg. 19:635, 1962.

12. COLLI, B. O. - Contribuição ao estudo do tratamento cirúrgico da neurocisticercose. Análise de 23 casos submetidos a derivacão extracraniana (derivação ventriculo-atrial e ventrículo-peritoneal). Tese. Ribeirão Preto, 1979.

13. COLLI, B. O.; MARTELLI, N.; ASSIRATI JR., J. A. \& MACHADO, H. R. Ventriculografia com Dimer-X em pacientes portadores de neurocisticercose com hipertensão intracraniana por hidrocefalia obstrutiva. No prelo. 
14. COLLI, B. O.; MARTeLLI, N.; ASSIRATI JR., J. A.; MACHADO, H. R. \& BELLUCI, A. - Tomografia computadorizada em pacientes portadores de neurocisticercose com hipertensão intracraniana por hidrocefalia obstrutiva: comparação com ventriculografia com Dimer-X. Arq. Neuro-Psiquiat. (São Paulo) 42:116, 1984.

15. CRUZ, M. E. - Ambulatory treatment of neurocysticercosis with praziquantel. Apresentado no Simpósio Internacional sobre Cisticercose, Angra dos Reis, maio 21, 1982.

16. DANZIGER, J. \& BLOCH, S. - Tapeworm cyst infestations of the brain. Clin. Radiol. 26:141, 1975.

17. DORFSMAN, J. - The radiologic aspects of cerebral cysticercosis. Acta. radiol. $1: 836,1963$.

18. FACURE, N. O.; FACURE J. J. \& NUCCI, A. - Aspecto tumoral da cisticercose intracraniana: abordagem cirúrgica. Arq. Neuro-Psiquiat. (São Paulo) 36:200, 1978.

19. FORJAZ, S. V. \& MARTINEZ, M. - Formas obstrutivas da neurocisticercose ventricular. Arq. Neuro-Psiquiat. (Săo Paulo) 19:16, 1961.

20. JUNG, R. C.; RODRIGUEZ, M. A.; BEAVER, P. C.; SCHENTHAL, J. E. \& LEVY, R. W. - Racemose cysticercus in human brain. Amer. J. trop. Med. Hyg. 30:620, 1981.

21. LAMAS, E. \& ESTEVEZ, J. - Dos casos de cisticercosis cerebral diagnosticados con tomografia axial computadorizada. Rev. clin. esp. 151:53, 1978.

22. LATOVITSKI, N.; ABRAMS, G.; CLARK, C.; MAYEUX, R.; ASCHERL JR., G. \& SCIARRA, D. - Cerebral cysticercosis. Neurology (Minneapolis) 28:838, 1978.

23. LEPE, A. \& CASTRO, M. - Radiologia de la cisticercosis cerebral. Neurocirugia (Santiago) 19:191, 1961.

24. LOMBARDO, L. - La cisticercosis cerebral em México. III. Diagnostico. Gac. med. mex. 118:4, 1982.

25. LOPES, P. G. - Contribuição ao estudo do tratamento cirúrgico da cisticercose da fossa craniana posterior. Tese. Londrina, 1970.

26. MARTELLI, N. - Tratamento cirúrgico da neurocisticercose. In F. C. Prado, J. A. Ramos \& J. R. Valle (eds.) - Atualização Terapêutica. Artes Médicas, são Paulo, 1981, pg. 730 .

27. MARTELLI, N.; COLLI, B. O.; ASSIRATI JR., J. A. \& MACHADO, H. R. Erosão óssea da base do crânio (regiões selar e para-selar) por cisticerco racemoso. Relato de caso. Arq. bras. Neurocirurg. 1:273, 1982.

28. MERVIS, B. \& LOTZ, J. W. - Computed tomography (CT) in parenchymatous cerebral cysticercosis. Clin. Radiol. 31:521, 1980.

29. PERCY, A. K. ; BYRD, S. E. \& LOGKE, G. E. - Cerebral cysticercosis. Pediatrics $66: 967,1980$.

30. PUPO, P. P.; REIS, J. B.; CARDOSO, W. \& SILVA, C. P. - Sobre a cisticercose encefálica. Estudo clínico, anátomo-patológico, radiológico e do líquido céfalo-raqueano. Arq. Assis psicop. Săo Paulo 10-11:3, 1945/1946.

31. ROBLeS CASTILLO, C. - Tratamiento médico de la cisticercosis cerebral. Salud publica mex. 23:443, 1981.

32. ROBLES, C. - Tratamiento médico de la cisticercosis cerebral. Gac. med. mex. 117:355, 1981.

33. ROBLeS, C. \& CHAVARRIA, M. C. - Presentación de un caso clínico de cisticercosis cerebral tratado médicamente con un nuevo fármaco: praziquantel. Salud publica mex. 21:603, 1979.

34. RODRfGUEZ, J. C.; GUTIERREZ, R. A.; VALDAS, O. D. \& DORFSMAN, J. F. - The role of computed axial tomography in the diagnosis and treatment of brain inflammatory and parasitic lesions: our experience in México. Neuroradiology $16: 458,1978$.

35. SCHENONE, H. - Resultados del tratamiento de la neurocisticercosis con praziquantel en Chile. Apresentado no Simpósio Internacional sobre Cisticercose, Angra dos Reis, maio 21, 1982.

36. SHIBATA, M. K.; BIANCO, F.; MOREIRA, F. A. \& ALMmIdA, G. M. - Forma tumoral da cisticercose cerebral: diagnóstico pela tomografia computadorizada. Arq. Neuro-Pqiquiat. (São Paulo) 38:399, 1980. 
37. SPINA-FRANÇA, A. - Neurocisticercose e praziquantel: avaliação dos resultados em 60 pacientes. Apresentado no Simpósio Internacional sobre Cisticercose, Angra dos Reis, maio 21, 1982.

38. SPINA-FRANCA, A. \& NOBREGA, J. P. S. - Neurocisticercose e praziquantel. Nota Prévia. Rev. paul. Med. 95:34, 1980.

39. SPINA-FRANÇA, A. \& NOBREGA, J. P. S. - Neurocisticercose e praziquantel: II - Avaliação dos resultados em 20 pacientes. Arq. Neuro-Psiquiat. (São Paulo) $39: 279,1981$.

40. STEPIEN, L. - Cerebral cysticercosis in Poland. Clinical symptons and operative treatment. J. Neurosurg. 19:505, 1962.

41. TAKAYANAGUI, O. M. - Aspectos clínicos da neurocisticercose. Análise de 500 casos. Tese. Ribeirăo Preto, 1980.

42. ZEE, C. S.; SEGALL, H. D.; MILLER, C.; TSAI, F. Y.; TEAL, J. S.; HIESHIMA, G.; AHMADI, J. \& HALLS, J. - Unusual neuroradiological features of intracranial cysticercosis. Radiology 137:397, 1980.

Departamento de Cirurgia, Ortopedia e Traumatologia (Disciplima Neurocimurgia) HC Campus - 14100 Ribeirão Preto, SP - Brasil. 